\title{
Habitateignungskartierung als Grundlage für die räumliche Planung von Pflege- und Entwicklungsmaßnahmen am Beispiel der Kreuzotter (Vipera berus)
}

\author{
Recording habitat-suitability as basis for spatial planning of man- \\ agement and development measures on the example of the common \\ European adder (Vipera berus)
}

\author{
Jörn Krütgen \\ Faunistisch-Ökologische Arbeitsgemeinschaft e.V. , c/o Institut für Natur- und Ressourcenschutz der Christian-Albrechts- \\ Universität zu Kiel, Olshausenstraße 75, 24118 Kiel, joern@foeag.de
}

\section{Schlüsselwörter:}

Schlange, Vipera berus, Habiateignung, Grünbrücke

\section{Keywords:}

snake, Vipera berus, habitat suitability, wildlife crossing

\begin{abstract}
Zusammenfassung
Die Habitateignung des Umfeldes der Grünbrücke Kiebitzholm an der Bundesautobahn A 21, Kreis Segeberg, Schleswig-Holstein, wurde für die Kreuzotter (Vipera berus) ermittelt. Dazu wurden Daten zur Populationstruktur, Raumnutzung sowie Habitatwahl gewonnen. Mit Hilfe eines Habitateignungsschlüssels wurde unter Verwendung eines Geographischen Informationssystems (GIS) die Habitateignung im näheren Umfeld der Querungshilfe räumlich analysiert und in Form einer Habitateignungskarte dargestellt. Es zeigt sich, dass das Modell die reale Verbreitung westlich der Autobahn sehr gut wiedergibt. Zudem wurde festgestellt, dass trotz des Fehlens der Art auf der Ostseite der A 21 in Teilbereichen eine hohe Habitateignung vorliegt. Habitatgestaltende Maßnahmen durch Schaffung von Korridoren beziehungsweise Trittsteinen im Umfeld der Grünbrücke und Maßnahmen zur Unterstützung der Population im Kiebitzholmer Moor könnten diese Flächen mittelfristig von der Kreuzotter besiedelt werden.
\end{abstract}

\footnotetext{
Abstract

The habitat-suitability of the wildlife crossing Kiebitzholm at the federal motorway A 21, district Segeberg, Schleswig-Holstein, and surrounding habitats was determined for the common European adder (Vipera berus). In addition to population-data, utilisation of space and habitat choice were estimated. Based on a habiat-suitability-index, a spatial analysis of the habitat-suitability of the study area was made useing a geographical information system (GIS). It was shown, that the actual distribution of the adder West of the motorway is well reflected by the model. According to the model different kinds of suitable habitats exist on the east of the motorway, although the adder is missing there. Therefore, it is proposed to implement corridors or stepping stones to support a recolonisation of these areas by the common European adder.
} 


\section{Einleitung}

Als Teil des Begleitmonitorings zum Bau der Grünbrücke Kiebitzholm im Zuge des Ausbaus der Bundestraße B 404 zur Autobahn A 21 wurde die Verbreitung der Kreuzotter (Vipera berus) erfasst. Außerdem wurde die Habitateignung der Lebensräume für die Art im Umfeld der Querungshilfe abgeschätzt. Ziel war die Erstellung einer GIS-gestützen Habitateignungskarte, die als Bewertungsgrundlage für die Akzeptanz der Grünbrücke durch die Kreuzotter dienen sollte. Sie sollte weiterhin Auskunft darüber geben, welche räumliche Planung von Pflege- und Entwicklungsmaßnahmen notwendig sind, um eine Erhöhung der Populationsgröße und einer Vernetzung potenzieller Lebensräume zu erreichen.

GIS-basierte Modelle zur Entwicklung von Schutz- und Pflegemaßnahmen für Reptilien, insbesondere für Schlangen, finden sich relativ selten. In der Regel handelt es sich um Arbeiten, die sich mit der potenziellen Verbreitung von Arten auf mittlerer Maßstabsebene beschäftigen (Guisan \& Hofer 2003 für die Schweiz, Santos et al. 2006 für Spanien, Krütgen 2009b für Schleswig-Holstein).

Auf Grundlage vorhandener Grundlagendaten war es möglich für den Raum Kiebitzholm ein einfaches Modell auf großer Maßstabsebene zu entwickeln welches die Größe, Lage und räumlichen Verteilung potenzieller Habitate aufzeigte. Diese wurden mit Geländedaten abgeglichen. $\mathrm{Zu}$ diesem Zweck erfolgte neben einer Habitateignungskartierung eine Erfassung der Raumnutzung und Habitatwahl der ansässigen Kreuzotterpopulation (Krütgen 2009a).

Die vorliegende Untersuchung soll am Beispiel der Kreuzotter die mögliche Verwendung von Habitatmodellen zur Planung von Pflege- und Schutzmaßnahmen auf lokaler Ebene, insbesondere unter Berücksichtigung von Querungshilfen, zeigen.

\section{Methoden}

\section{Untersuchungsgebiet}

Das Untersuchungsgebiet liegt im direkten Umfeld der Grünbrücke Kiebitzholm (Gemeinde Negernbötel, Kreis Segeberg). Es wird durch einen etwa $2 \mathrm{~km}$ großen Radius, ausgehend vom Zentrum der Grünbrücke, abgegrenzt. Das Untersuchungsgebiet umfasst weite Teile des nadelholzdominierten Staatsforstes Kiebitzholm. In und am Rand des Staatsforstes existieren auf beiden Seiten der A 21 Reste ehemals größerer Sand- trockenrasen und - heiden. Vor allem in den nördlichen und westlichen Randbereichen befinden sich zudem größere Feuchtgrünland- und Moorbereiche. Im Südwesten existieren mehrere ehemalige Abbaugruben. Das Gebiet wird von der A 21 von Nordwesten nach Südosten zerschnitten. Die Grünbrücke befindet sich in der Nordhälfte des Kiebitzholmer Forstes.

\section{Habitateignungsschlüssel}

Anhand der Ergebnisse anderer Untersuchungen (z.B. Schiemenz 2004, Völkl \& Thiesmeier 2002), Expertengesprächen und eigenen Beobachtungen aus verschiedenen Biotopen in Schleswig-Holstein wurde ein Habitateignungsschlüssel für die Kreuzotter erstellt. Der Schlüssel umfasst sieben Parameter: Biotoptyp (nach LANU 2003), Flächengröße, Bodenart, Exposition, Gehölzbedeckung, Strukturvielfalt sowie Nahrungsverfügbarkeit. Dabei wurden die Ausprägungen jedes Parameters den Skalensstufen "+ + “, " + ", " 0" oder "-“" zugeordnet und aufsummiert, so dass maximal $14+$ aber auch negative Werte erreicht werden konnten. Mit Ausnahme der Flächengröße wurden sämtliche Parameter im Gelände erhoben. Die Flächengröße wurde im GIS errechnet. Es wurde nicht nach saisonalen Teilhabitaten unterschieden. Eine Eignung beispielsweise als reiner Sommerlebensraum floss bei der Bewertung des Biotoptyps mit ein.

\section{Habitateignungskarte}

Auf Basis der Biotoptypen-Nutzungskartierung (BTNK) des LLUR aus dem Jahr 2000 wurde eine Habitateignungskarte für das Untersuchungsgebiet erstellt. Bei der BTNK handelt es sich um eine landesweite CIRLuftbild - gestützte Kartierung der Biotope sowie Nutzungsformen in Schleswig-Holstein (Brand et al. 2002). Die Daten lagen als Flächendaten in Form einer shapeDatei vor und konnten mit Hilfe eines GIS (ArcMap 9.3) weiterverarbeitet werden. Die BTNK wurde durch die Ergebnisse einer Biotopkartierung von C. Dolnik aus dem Jahre 2006 ergänzt. Die Bewertung erfolgte anhand des Biotoptyps, analog zum ersten Parameter des Habitateignungsschlüssels. Auch wurden seine Skalenstufen übernommen (s.o.). Anhand der gewonnenen Daten mit Hilfe des Habitateignungsschlüssels wurde die Auflösung der Habitateignungskarte erhöht. Zu die- 
sem Zweck wurden zunächst die Skalenniveaus einander angepasst, indem die Ergebnisse der Bewertung anhand des Schlüssels auf die vierstufige Skalierung der Biotoptypenbewertung umgerechnet (vgl. Tab. 1) und die Ergebnisse anschließend verschnitten wurden.

\section{Erfassung der Kreuzotter}

In den Jahren 2006, 2008 und 2010 wurden die Reptilien im Umfeld der Grünbrücke erfasst (Winkler 2016). Die Erfassung erfolgte durch Absuchen von Sonnenplätzen im Frühjahr und im Herbst. Neben den von Winkler kontrollierten Probeflächen wurden im September 2008 potenzielle Sonnenplätze am Südostrand des Kiebitzholmer Moores annährend vollständig nach Kreuzottern abgesucht. Zum Nachweis der Kreuzotter wurden in potenziellen Habitaten zusätzlich künstliche Verstecke ausgelegt.

Bei den Begehungen entdeckte Kreuzottern wurden gefangen und die Kopfoberseiten zur individuellen Erkennung fotografiert (vgl. Lenders 2000) sowie Alter und Geschlecht aufgenommen. Anschließend wurde der Fundort mittels GPS-Empfänger ermittelt und die Fundpunkte in ein Geographisches Informationssystem (GIS) eingespeist. Darüber hinaus wurden sie dem Landesamt für Landwirtschaft, Umwelt und Ländliche Räume (LLUR) gemeldet und dort in das Arten- und Fundpunktkataster Schleswig-Holstein (AFK) aufgenommen. Im Gegenzug wurden die eigenen Erhebungen durch Fundmeldungen aus dem AFK ergänzt. Auf Basis dieser Daten konnte eine Verbreitungskarte der Kreuzotter für das Untersuchungsgebiet erstellt werden. Die gewonnenen Daten dienten zudem als Kontrolle für die Habitatbewertung.

\section{Ergebnisse}

\section{Habitatbewertung}

Tabelle 1 zeigt die Größe und Verteilung der mit Hilfe des Habitatschlüssels bewerteten Flächen im Untersuchungsgebiet. Rund 30 \% der Gesamtfläche wird von Biotopen eingenommen, die für die Art ungeeignet sind. Hierbei handelt es sich vor allem um Äcker und Forste aber auch um Verkehrsflächen. Den größten Flächenanteil machen Flächen ohne Eignung als Kreuzotterlebensraum aus. Deutlich geringer ist der Anteil an Flächen mit Eignung und sehr guter Eignung. Deren durchschnittliche Flächengröße ist ebenfalls deutlich geringer.

Die räumliche Analyse ergibt, dass sich die größten zusammenhängenden Bereiche mit hoher Habitateignung im Nord-Westen des Untersuchungsgebietes befinden (Abb. 1). Hier stehen der Kreuzotter auf nahezu 100 ha verschiedene potenzielle Lebensräume zur Verfügung. Diese Flächen umfassen Restmoorkomplexe, Heideflächen, Extensivweiden, Bracheflächen, diverse Wegränder sowie schüttere Birkenwälder auf Moorstandorten und deren Säume.

Auf der Ostseite stehen der Kreuzotter ebenfalls potenzielle Habitatfläche zur Verfügung. Die Flächen sind insgesamt jedoch kleiner und liegen, vor allem im Fall der besonders gut geeigneten Flächen, stärker zerstreut und isoliert durch Flächen ohne Habitateignung (i.d.R. Wald oder Äcker). Trotzdem eignen sich bereits einige Flächen für eine (Wieder-)besiedlung durch die Kreuzotter, bzw. könnten der Art als Trittstein in einer Ausbreitungsachse, beispielsweise zum System der Faulen Trave oder dem Trentmoor im Süd-Osten des Untersuchungsgebietes, dienen.

Tab. 1: Größe und Verteilung der Flächen nach Eignung als Kreuzotterhabitat.

\begin{tabular}{lllll}
$\begin{array}{l}\text { Eignung } \\
\text { Karte }\end{array}$ & $\begin{array}{l}\text { Eignung } \\
\text { Schlüssel }\end{array}$ & $\begin{array}{l}\text { Flächensumme } \\
\text { in ha }\end{array}$ & $\begin{array}{l}\text { Flächensumme } \\
\text { in } \%\end{array}$ & $\begin{array}{l}\text { durchschnittliche } \\
\text { Flächengröße in ha }\end{array}$ \\
\hline k.A. & 1023,8 & 52,09 & 68,25 \\
- & $-/ 0$ & 581,19 & 29,57 & 25,26 \\
0 & $1+-4+$ & 101,93 & 5,19 & 20,39 \\
+ & $5+-7+$ & 142,52 & 7,25 & 3,39 \\
++ & $8+-10+$ & 115,95 & 5,90 & 2,76 \\
\hline gesamt & & 1959,39 & 100,00 & 24,01
\end{tabular}




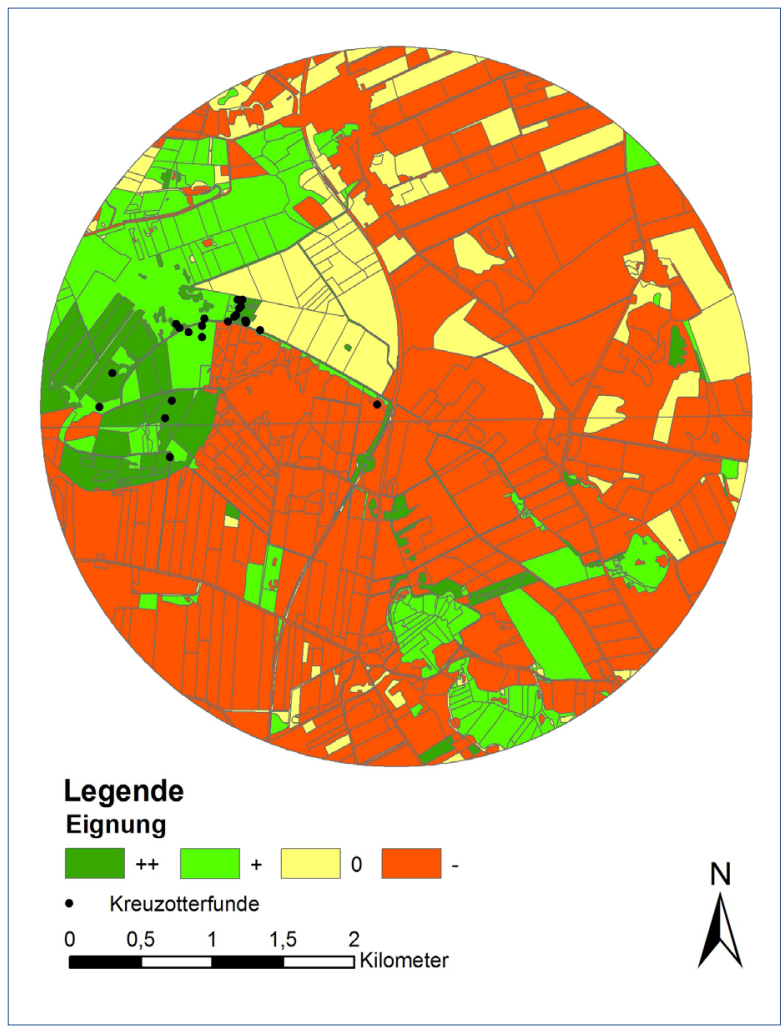

Abb. 1: Habitateignung auf Basis der Biotoptypen-Nutzungskartierung, verändert nach Dolnik (2007) und eigenen Erhebungen (2008); Kreuzotterfunde (AFK, Stand: 2008).

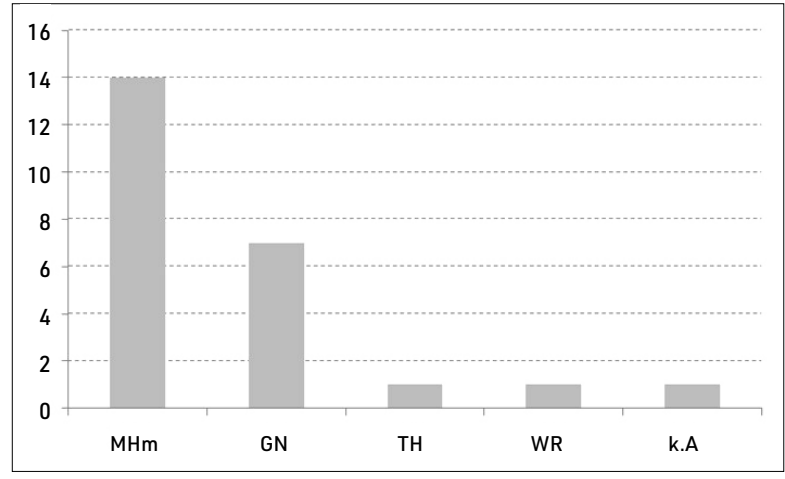

Abb. 2: Anzahl Kreuzotterfundorte (Stand 2008) nach Biotoptypenschlüssel LANU 2003. MH: Hoch- Übergangsmoore, GN: Seggenund Binsenreiches Feucht- und Nassgrünland, TH: Zwergstrauchheiden, WR: Waldrand, k.A.: keine Angabe.

Die meisten Kreuzotter- Fundorte (Angaben des AFK, Stand 2008) befanden sich im Randbereich des ehemaligen Hochmoores (i.d.R. Pfeifengrasstadien), gefolgt von seggen- und binsenreichem Feucht- und Nassgrünland. Ein Fund gelang an einem Heidestandort sowie an einem Waldrand, ein weiterer Fundort besitzt keine Biotopzuordnung.
Die meisten Individuen wurden auf einer von Flatterbinsen und Pfeifengras bestandenen Feuchtgrünlandbrache und einer sich anschließenden von Pfeifengras dominierten Restmoorfläche im Norden des Untersuchungsgebietes beobachtet. Darüber hinaus fanden sich Kreuzotter im gesamten Bereich des Moor- und Heidekomplexes westlich des Kiebitzholmer Forstes inklusive der hier gelegenen Extensivweide (Abb. 2).

Auffällig ist die gute Wiedergabe der realen Fundorte von Kreuzottern durch die Habitateignungskarte (Abb. 1). Von den 24 Fundorten, die das AFK (Stand 2008) für das Gebiet aufweist, lagen zwölf in Bereichen mit sehr guter Habitateignung, zehn in solchen mit Eignung. Es wurde jeweils nur ein Tier in Bereichen mit neutraler oder fehlender Eignung gefunden. Betrachtet man nur die Daten der BTNK, ohne eine Validierung durch die eigene Erhebung (Krütgen 2009), so fanden sich vier Fundorte auf ungeeeigneten, drei auf neutralen, sieben auf geeigneten und zehn Fundorte auf sehr gut geeigneten Flächen. Das heißt, dass rund $42 \%$ aller Fundorte in Bezug auf die Eignung abweichen.

\section{Aktuelle Verbreitung}

Insgesamt konnten im Untersuchgungsraum 32 Fundorte der Kreuzotter ermittelt werden (Abb. 1), von denen 20 aus eigenen Erhebungen und 12 aus den Daten des AFK stammten. Die Fundorte konzentrierten sich auf die südöstlichen Teile des Kiebitzholmer Moores. An dem Fundort nahe der Autobahn am nördlichen Waldrand (Abb. 3) konnte die Art bislang nicht wieder bestätigt werden.

Im Ostteil des durch die A 21 getrennten Untersuchungsgebietes konnte die Kreuzotter nicht nachgewiesen werden. Auch weist das AFK (Stand 2010) keine Funde in diesem Bereich auf.

\section{Diskussion}

\section{Habitatbewertung}

Die Habitatbewertung ergab, dass im Gebiet relativ große Flächen liegen, die für eine Besiedlung durch die Kreuzotter in Frage kommen. Diese finden sich vor allem im Kiebitzholmer Moor und dessen Randflächen im Nord-Westen des Untersuchungsgebietes. Aufgrund der sehr guten Wiedergabe der realen Fundorte aus dem AFK (Stand 2008) durch die Habitateignungskarte 


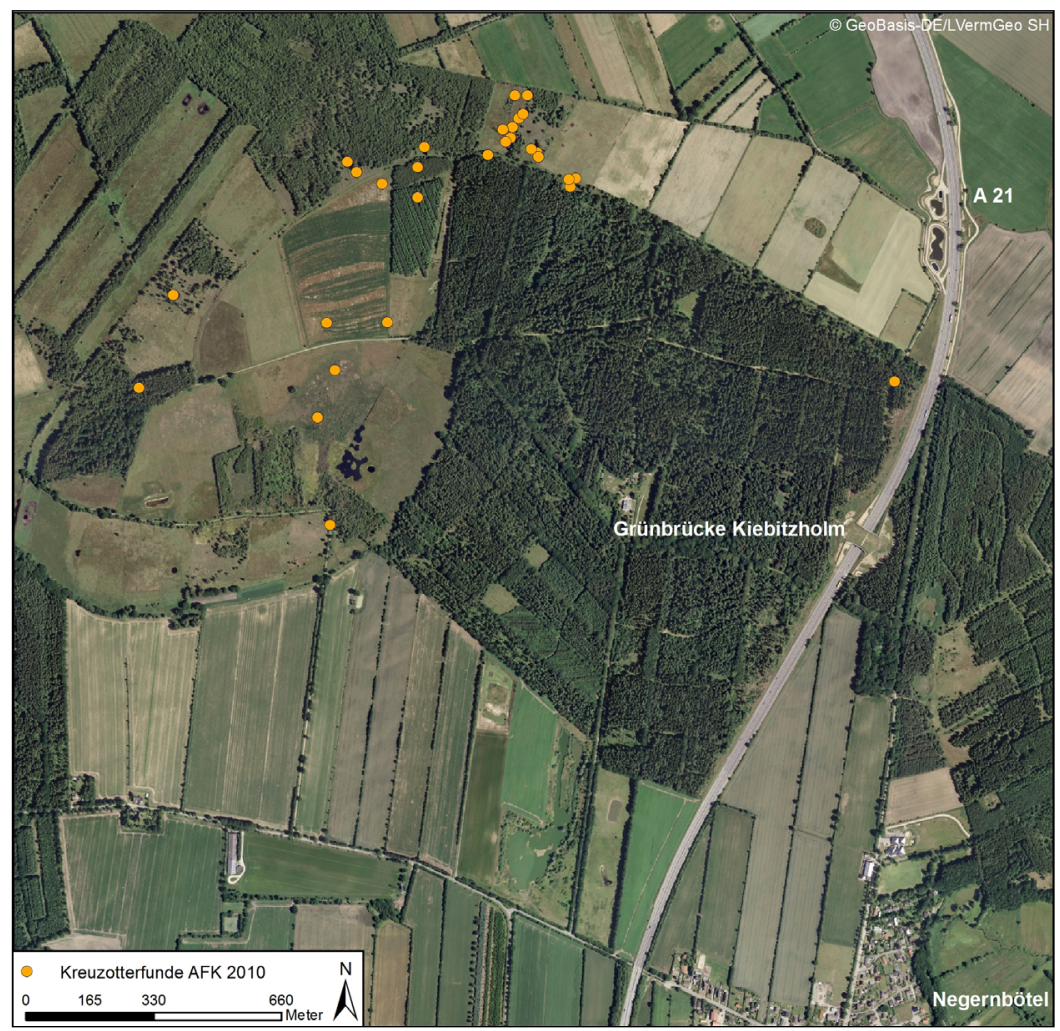

Abb. 3: Fundorte der Kreuzotter im Untersu chungsgebiet nach eigenen Daten und Daten des Arten- und Fundpunktkataster SchleswigHolstein (AFK) (Stand: 2010). Kartengrundlage: DOP 40 2009, @ LVermGeo SH 2011. erscheint eine fehlerhafte Einschätzung der Habitateignung auch östlich der Autobahn, trotz fehlender Nachweise der Art, als unwahrscheinlich. Hier liegt eine Vielzahl an kleineren Flächen, die zum Teil bereits in einem Verbund stehen und einen potenziellen Lebensraum für die Kreuzotter darstellen. Die hohe Kartierungsdichte im Gebiet seit 2006 (Winkler \& Krütgen 2010) spricht dafür, dass die Kreuzotter im östlichen Teil des Untersuchungsgebietes mit hoher Wahrscheinlichkeit nicht übersehen wurde.

Diese Güte wurde jedoch nur durch die Validierung der Habitateignungskarte erreicht, da die Daten mit Hilfe des Habitatschlüssels gesammelt wurde n. Eine rein auf Geodaten basierende Karte (z.B. BTNK 2000) erlangt nicht die nötige Schärfe für lokale Maßnahmenplanungen. Sie eignen sich besonders auf regionaler Ebene zur Ermittlung von Vorrangräumen für Maßnahmen (Krütgen 2009).

Die validierte Habitateignungskarte eignet sich dagegen gut für die Planung von lokalen Pflege- und Entwicklungsmaßnahmen zum Schutz der Kreuzotter. Ebenfalls kann sie als Grundlage für die räumliche Planung von Maßnahmen für eine Wiederausbreitung der Art im Untersuchungsgebiet dienen.

\section{Wiedervernetzung geeigneter Habitate}

Unter Beachtung der Habitateignungskarte erscheint eine Querung der A 21 bei Schaffung von Habitatkorridoren möglich. Der Verbund mit Hilfe des Ottertunnels ist jedoch als problematisch anzusehen, da in seiner Umgebung auf der Ostseite ausgedehnte Flächen mit ackerbaulicher Nutzung liegen und eine Verbesserung der Situation von der Bereitschaft der Landeigner zum Verkauf / Verpachten der Flächen abhängen würde.

Erfolgversprechender erscheint der Verbund über die Grünbrücke, die selbst bereits eine höhere Habitateignung aufweist. Diese ist auf die eingebrachten Strukturen wie Totholzhaufen und Baumstubben oder auch die niedrigwüchsige Vegetation im Zusammenspiel mit offenen Bodenstellen zurückzuführen. Es muss jedoch damit begonnen werden, die Zugänglichkeit zu erleichtern. Zurzeit behindert der Fichtenforst zu beiden Seiten der Autobahn einen Habitatverbund.

Betrachtet man die Habitateignungskarte, so ergeben sich westlich der Grünbrücke zwei mögliche Achsen zur Vernetzung des Bauwerkes mit der Population im Kiebitzholmer Moor. Zum einen könnte der südliche Waldrand aufgelichtet werden. Derzeit befindet sich hier ein aufgegebener Wirtschaftsweg, der durch eine 
Reihe von Eichen beschattet wird. Um Konflikte mit anderen Zielen des Naturschutzes zu vermeiden, sollte dabei auf ein Fällen dieser Eichen verzichtet werden und stattdessen der Waldrand dahinter, ggf. als windgeschützte Einbuchtungen, aufgelichtet werden. Ein Problem könnte jedoch die ackerbauliche Nutzung der sich südlich anschließenden Flächen mit sich bringen. Es sollte ein Puffer eingerichtet werden, um einen übermäßigen Nährstoffeintrag abzumildern. Die zweite Option könnte die Aufwertung des nördlichen Waldrandes darstellen. Dieser besitzt derzeit eine sehr gute Strukturvielfalt. Hier ist einzig die Nord-Exposition als negativ zu bewerten. Um dies auszugleichen, sollten die angrenzenden Grünlandflächen extensiviert werden, um auch hier für eine ausreichende Strukturvielfalt zu sorgen und eine Besiedlung der Fläche durch die Kreuzotter zu begünstigen.

In beiden Fällen muss der östliche Waldrand aufgewertet werden. Auch wenn die relative Offenheit als positiv einzuschätzen ist, so muss gewährleistet werden, dass das Gebiet nicht durch Arten wie die Traubenkirsche (Prunus serotina) verbuscht.

Auf der Ostseite der A 21 muss ebenfalls ein Fichtenforstriegel überwunden werden, um die nächstgelegene geeignete Fläche zu erreichen. Eine Option stellt dabei die Auflichtung des Waldes Richtung Süden dar. Entweder könnte ein ehemaliger Redder, der Richtung Osten verläuft, oder der Waldrand, der jedoch hier ohne Übergang an einen Acker grenzt, aufgelichtet werden. Die Einrichtung eines Puffers wäre auch hier nötig. Die parallel zur Autobahn verlaufende Nebenstraße weist eine nur geringe Verkehrsdichte auf, so dass diese nicht als Barriere zu werten ist. Ein Großteil der Straßenböschungen kann bereits jetzt für einen Verbund von (Teil-)Habitaten dienen. Trotzdem sollten auch hier Aufwertungsmaßnahmen durchgeführt werden. So wird sichergestellt, dass verschiedene Flächen, die eine positive Habitateignung aufweisen (wie beispielsweise die ehemalige Abbaugrube in der Nähe der Grünbrücke) von der Kreuzotter erreicht werden und in einen funktionalen Verbund gesetzt werden. Dadurch könnte eine Metapopulation entstehen, in der die bestehende Population als Donatorpopulation fungieren könnte (Settele et al.1996). Die Vorraussetzungen hierfür wären eine regelmäßige Querung des Bauwerks durch Kreuzottern.

Neben der Schaffung von Korridoren können zudem Maßnahmen zur Optimierung der Habitateignung im Randbereich des Kiebitzholmer Moores zweckdien- lich sein. Ein Anstieg der Anzahl an Tieren erhöht die Tendenz einer Abwanderung. Dies könnte durch eine höhere Nahrungsverfügbarkeit erreicht werden. Ein möglicher Ansatz wäre die Schaffung von Laichgewässern für Braunfrösche (Völkl \& Hansbauer 2010), die als Nahrung juveniler Kreuzottern das Anwachsen der Population fördern.

Die nächstgelegene Population der Kreuzotter östlich der A 21 befindet sich im Tarbeker Moor, etwa vier Kilometer nordöstlich der Grünbrücke. Langfristig wäre es erstrebenswert die Populationen im Kiebitzholmer Moor mit dieser (wieder) zu vernetzten. Die Grünbrücke Kiebitzholm und gegebenenfalls der nahe gelegene Ottertunnel könnten eine Querung der Autobahn ermöglichen. Nach erfolgreicher Hinterlandanbindung der Querungshilfen könnten gezielt Habitatbausteine und Korridore im Zuge des vom Bundesamt für Naturschutz geförderten Entwicklungs- und Erprobungsprojektes Holsteiner Lebensraumkorridore oder durch den gezielten Einsatz von Ausgleichsmitteln des Kreises geschaffen werden. Hiervon würden nicht nur die Kreuzotter, sondern auch andere schutzbedürftige Arten profitieren.

\section{Danksagung}

Ich danke Christian Winkler, Bordesholm, und PD Dr. Heinrich Reck, Schwentinental, für die kritische Durchsicht des Manuskriptes, sowie Lasse Christiansen, Kiel, für die Unterstützung bei der Übersetzung der Kurzfassung ins Englische.

\section{Autor}

\section{Jörn Krütgen}

Jörn Krütgen studierte in Kiel Geographie. Landschaftsökologische Themen mit Schwerpunkt des faunistischen Artenschutzes begleiteten fortan seine Arbeit. Die Herpetofauna bildet hierbei sein Hauptinteressensgebiet. Seit 2017 ist er Mitarbeiter der Oberen Naturschutzbehörde des Landes Schleswig-Holstein in der Abteilung Naturschutz und Forst. 


\section{Literatur}

Brand J, Ehlers M, Möller M (2002) Abschlussbericht des Projektes "Fortschreibung der Biotop- und Nutzungstypenkartierung mit multispektralen Scannerdaten" im Auftrag des Landesamtes für Natur und Umwelt des Landes Schleswig-Holstein (LANU). Vechta.

Dolnik C (2007) Vegetationsökologischer Teil zum Projekt „Bioökologische Wirksamkeit der Grünbrücke Kiebitzholm". Projektbericht 2006. Unveröffentlichtes Gutachten. Kiel.

Guisan A, Hofer U (2003) Predicting reptile distributions at the mesoscale: relation to climate and topography. J Biogeogr 30:1233-1243.

Krütgen J (2009a) Untersuchungen zur Verbreitung der Kreuzotter (Vipera berus) in Schleswig-Holstein als Grundlage für ein GISbasiertes Monitoring. Unveröff. Diplomarbeit am Geographischen Institut der Christian-Albrechts-Universität zu Kiel.

Krütgen J (2009b) GIS-basierte Analyse der aktuellen Verbreitung der Kreuzotter (Vipera berus) in Schleswig-Holstein. RANA 10:74-81.

Krütgen J (2011) Schaffung von Lebensraumstrukturen für Reptilien durch Schalenwild. Rana 12:63-65.

LANU, Landesamt für Natur und Umwelt des Landes Schleswig-Holstein (2003): Standardliste der Biotoptypen in Schleswig-Holstein, 2. Fassung. Flintbek

Lenders T (2000) Merkmethoden bij de herpetofauna. Patronen van kopschilden als individuele herkenning bij de adder. RAVON 7:13-18

Santos X, Brito JC, Sillero N, Pleguezuelos JM, Llorente GA, Fahd S, Parellada $X$ (2006) Interferring habitat-suitability areas with ecological modelling techniques and GIS: A constribution to asses the conservation status of Vipera latastei. Biol. Conserv. 130:416-425.

Schiemenz H (2004) Die Kreuzotter Vipera berus, 4., unveränderte Auflage, Nachdruck der 3. Auflage von 1995. Westarp WissenschaftenVerlagsgesellschaft $\mathrm{mbH}$, Hohenwarsleben.

Settele J, Henle K, Bender C (1996) Metapopulation im Biotopverbund: Theorie und Praxis am Beispiel von Tagfaltern und Reptilien. Zeitschrift für Ökologie und Naturschutz 5:187-206.

Völkl W, Thiesmeier B (2002) Die Kreuzotter. Ein Leben in festen Bahnen? Laurenti-Verlag, Bielefeld.

Völkl W, Hansbauer G (2010) Die Kreuzotter in Bayern. Erfolgreicher Artenschutz. Bayerisches Landesamt für Umwelt, Augsburg.

Winkler C, Krütgen J (2010) Zur Bedeutung der Grünbrücke Kiebitzholm im Kreis Segeberg (Schleswig-Holstein) für die Amphibien- und Reptilienfauna Ergebnisse aus den Jahren 2006 bis 2009. Rana 11:56-62.

Winkler C (2016) Zur Bedeutung der Grünbrücke Kiebitzholm für Reptilien. Faun. Ökol. Mitt 9:431-442.

\section{Open Access}

> Der Artikel ist unter der Creative-Commons-Lizenz Namensnennung 4.0 International veröffentlicht. Den Vertragstext finden Sie unter: https://creativecommons.org/licenses/by/4.0/deed.de. Bitte beachten Sie, dass einzelne, entsprechend gekennzeichnete Teile des Artikels von der genannten Lizenz ausgenommen sein bzw. anderen urheberrechtlichen Bedingungen unterliegen können. 